\title{
Multimembrane dot-blotting: a cost-effective tool for proteome analysis
}

\author{
Mikhail M. Galperin ${ }^{1}$, June L. Traicoff ${ }^{1}$, Arun Ramesh${ }^{1}$, Wendy J. Freebern², Cynthia M. Haggerty², \\ Dan-Paul Hartmann ${ }^{3}$, Michael R. Emmert-Buck², Kevin Gardner², and Vladimir Knezevic ${ }^{1}$
}

BioTechniques 36:1046-1051 (June 2004)

The molecular profiles of protein expression from hundreds of cell lysates can be determined in a high-throughput manner by using fluorescent bead technologies, enzyme-linked immunosorbent assays (ELISAs), and protein microarrays. Although powerful, these tools are costly and technically challenging and thus have limited accessibility for many research groups. We propose a modification of traditional dot blotting that increases throughput of this approach and provides a simple and cost-effective technique for profiling multiple samples. In contrast to traditional blotting that uses a single membrane, we introduce blotting onto a stack of novel, thin, sieve-like membranes. These membranes have a high affinity for binding proteins, but have a lower capacity of protein binding compared to traditional (nitrocellulose) membranes. We compare the linear binding capacity and variability of these novel membranes with nitrocellulose membranes. Also, we describe the use of these membranes in a multilayer dot blot format for profiling mitogenmediated signal transduction pathways in T cells.

\section{INTRODUCTION}

High-throughput protein screening of cell lines is a standard method of selecting compounds to be developed into therapeutic agents. Technologies available for high-throughput studies of protein expression include enzyme-linked immunosorbent assay (ELISA)-related systems and protein arrays (1-4). However, most of these approaches are costly and technically challenging, thus limiting their use for smaller research groups. To provide a simple, cost-effective alternative approach for protein expression profiling in multiple samples, a multilayer multiwell plate dot blotting system (MLDot) was developed. MLDot uses a layered membrane stack in place of a single nitrocellulose membrane allowing preparation of up to 10 membranes from the samples arrayed in a single 96well plate. Thus, MLDot can be used in a typically equipped laboratory and provides the ability to simultaneously profile multiple proteins in multiple samples.

MLDot technology is based on protein function and identification layered membranes (P-FILM) sieve-like membranes $(5,6)$. Their thinness (approximately $10 \mu \mathrm{m}$ as compared to approximately $100 \mu \mathrm{m}$ of conventional membranes), high affinity, and low protein binding capacity $\left(10-30 \mathrm{ng} / \mathrm{cm}^{2}\right)$ allow samples to be transferred simultaneously to multiple individual layers (7).

Using MLDot technology is relatively simple and straightforward. Protein lysates contained in a 96-well plate are trans- ferred through the P-FILM stack using a vacuum manifold. The amounts of both total protein and a specific protein of interest are then quantitated for each sample on each membrane by using antibodies/reagents coupled with fluorescent tags.

One potential application of MLDot is high-throughput proteomics profiling of drug-treated cells. This would allow identification of the signaling protein targets of a drug in a given cell line. This assay would also complement existing assays used to identify drugs that cause growth retardation or cytotoxicity against a panel of tumor cell lines. Since MLDot can simultaneously capture proteins from multiple samples on multiple membranes, the expression and activity of several signaling mediators in response to numerous treatments can be quantitatively measured in a single experiment. In this report, by using MLDot, we measured the response of T cells to an assortment of treatments.

\section{MATERIALS AND METHODS}

\section{Extraction of Proteins}

Jurkat cell line was cultured in RPMI media with $10 \%$ fetal calf serum (FCS) at $37^{\circ} \mathrm{C}$, lysed with Sample Lysis Buffer (20/20 GeneSystems, Rockville, MD USA), and centrifuged at $16,000 \times g$. The supernatant was then collected and stored at $-20^{\circ} \mathrm{C}$.

\footnotetext{
120/20 GeneSystems, Rockville, ${ }^{2}$ National Institute of Cancer, Bethesda, MD and

${ }^{3}$ Georgetown University Hospital, Washington, D.C., USA
} 


\section{Transfer of Proteins by MLDot}

Lysates containing $15 \mu \mathrm{g}$ of total protein ( $100 \mu \mathrm{L}$ volume) were loaded (or lysed directly) in each well of a 96-well plate and transferred onto a five-membrane stack of P-FILM membranes (20/20 GeneSystems) by vacuum transfer through the Bio-Dot ${ }^{\circledR}$ Microfiltration Apparatus (Bio-Rad Laboratories, Hercules, CA, USA). After transfer, the membranes were rinsed in deionized water, separated, and air-dried.

\section{Detection of Total Protein}

Biotinylation was performed by incubating membranes in $0.001 \%$ EZ -Link ${ }^{\mathrm{TM}}$ Sulfo-NHS-Biotin (Pierce Chemical, Rockford, IL, USA) solution in $1 \times$ phosphate-buffered saline (PBS) for $10 \mathrm{~min}$, followed by three washes ( $3 \mathrm{~min}$ each) in TBST buffer ( $50 \mathrm{mM}$ Tris, $150 \mathrm{mM} \mathrm{NaCl}, 0.05 \%$ Tween $^{\circledR 20}$ ), air-drying, and incubation with a streptavidin-Cy ${ }^{\mathrm{TM}} 5$ conjugate.

\section{Detection of Specific Protein}

Membranes were incubated overnight at $4^{\circ} \mathrm{C}$ in TBST plus $0.1 \%$ bovine serum albumin (BSA) solution of antibodies against the following proteins: phospho-tyrosine (1:1000;
Cell Signaling, Beverly, MA, USA), c-Myc, (1:500; NeoMarkers, Fremont, CA, USA), c-Raf -1 (1:500; BD Transduction Laboratories/BD Biosciences, San Diego, CA, USA). After three washes ( 3 min each) in TBST buffer, membranes were incubated for $1 \mathrm{~h}$ at room temperature in fluorescein goat-anti-mouse immunoglobulin $\mathrm{G}(\mathrm{IgG})$ (1:1000; Molecular Probes, Eugene, OR USA), washed three times (3 $\mathrm{min}$ each) in TBST buffer, and air-dried.

\section{Fluorescent Detection of Protein Signal}

Membranes were scanned on the Typhoon ${ }^{\circledR}$ scanner (Amersham Biosciences, Piscataway, NJ, USA). Signal intensity was determined by ImageQuant ${ }^{\circledR}$ software (Amersham Biosciences), and data were graphed and analyzed by Microsoft ${ }^{\circledR}$ Excel $^{\circledR}$ and JMP (SAS Institute, Cary, NC, USA).

\section{Quantitation and Normalization of Protein Signal}

For total protein on each membrane, the signal intensity of the uppermost left sample was arbitrarily set at 1.0. The signal intensity of each of the other 95 samples was then normalized to this reference sample. The intensity of each specific protein signal was normalized to its corresponding total protein signal. 
Table 1. Variability in Signal Intensity for Proteins Transferred to P-FILM or Nitrocellulose Membranes

\begin{tabular}{|c|c|c|c|c|}
\hline & Total F & Protein & Specific & Proteins \\
\hline & $\begin{array}{c}\text { Intermembrane } \\
\text { Variability }\end{array}$ & $\begin{array}{c}\text { Intramembrane } \\
\text { Variability } \\
(\%)\end{array}$ & $\begin{array}{c}\text { Intermembrane } \\
\text { Variability }\end{array}$ & $\begin{array}{c}\text { Intramembrane } \\
\text { Variability } \\
(\%)\end{array}$ \\
\hline P-FILM & 1.01 & 23 & 1.87 & 19.8 \\
\hline NC & 0.64 & 17 & 1.58 & 19.0 \\
\hline $\begin{array}{l}\text { Intermem } \\
\text { nal intens } \\
\text { P-FILM n } \\
\text { variability } \\
\text { to membi } \\
\text { ability (th } \\
\text { identifica }\end{array}$ & $\begin{array}{l}\text { ane (through the stac } \\
\text { of total and specific } \\
\text { nbranes by MLDot or } \\
\text { the maximum throug } \\
\text { e } 1 \text {, and intramembr } \\
\text { tandard deviation as } \\
\text { layered membranes layed }\end{array}$ & $\begin{array}{l}\text { k) and intramembrane } \\
\text { proteins were calculat } \\
\text { to nitrocellulose (NC } \\
\text { h-the-stack variability } \\
\text { ane variability design } \\
\text { a percentage of mea }\end{array}$ & $\begin{array}{l}\text { (across the membran } \\
\text { following transfer of } \\
\text { membranes by dot bl } \\
\text { as a fold difference of } \\
\text { tes average across-th } \\
\text { intensity). P-FILM, }\end{array}$ & $\begin{array}{l}\text { e) variability in sig- } \\
15 \mu \mathrm{g} \text { of protein to } \\
\text { lot. Intermembrane } \\
\text { intensity compared } \\
\text { ee-membrane vari- } \\
\text { rotein function and } \\
\text { g system. }\end{array}$ \\
\hline
\end{tabular}

\section{Isolation and Mitogen/Drug Treatment of Lymphocytes}

Primary blood lymphocytes were obtained as previously described (8) and treated with the indicated mitogen/drug combinations at the following concentrations: $50 \mathrm{ng} / \mathrm{mL}$ phorbol myristate acid (PMA; Sigma, St. Louis, MO, USA), $720 \mathrm{ng} / \mathrm{mL}$ ionomycin (Calbiochem, San Diego, CA, USA), $1.5 \mu \mathrm{g} / \mathrm{mL}$ anti-CD28 (Research Diagnostics, Flanders, NJ, USA), 1:1000 dilution anti-CD3 ascites fluid (ATCC, Manassas, VA, USA) and $40 \mathrm{ng} / \mathrm{mL}$ insulin-like growth factor 1 (IGF-1) (Research Diagnostics). After a 5-h incubation at $37^{\circ} \mathrm{C}$, cells were harvested, washed twice with PBS, and lysed in a modified radioimmunoprecipitation (RIPA) buffer.

\section{RESULTS AND DISCUSSION}

\section{Visualization of Total Protein on P-FILM Membranes}

Our first goal was to find a simple and reliable method for quantitation of total protein on P-FILM membranes in order to normalize for differences in protein amounts between samples and thereby eliminate the need for initial assaying of protein concentrations. We developed a novel approach of total protein visualization based on attaching biotin residues to protein by using N-hydroxysuccinimide (NHS) reactive ester chemistry and visualizing these biotin tags with streptavidin-Cy5 conjugates $(9,10)$. After transfer, proteins were first biotinylated directly on the membrane. Membranes were then incubated in primary antibody. Following a washing step, membranes were simultaneously incubated with a streptavidin-Cy5 conjugate and secondary antibody. We find biotinylation to be advantageous over ubiquitous protein staining because of better antibody binding to the epitopes on biotinylated protein than to the epitopes on stained proteins. Also, biotinylation decreases membrane processing time by allowing concurrent detection of total and specific protein by two-channel scanning on a fluorescent scanner.

\section{Variability Within and Between P-FILM Membranes}

We next measured intramembrane variability (the distri- bution of signal intensities between samples on a single membrane) and intermembrane variability (the average difference of signal intensity between membranes in the stack as compared to the first one). Five, ten, and twenty micrograms of Jurkat lysates were transferred one time onto a five-membrane P-FILM stack by MLDot or five times to five nitrocellulose membranes by traditional dot blot. The levels of c-Myc and c-Raf were measured by incubating the individual membranes with corresponding antibodies and visualizing complexes with fluorescein isothiocyanate (FITC)-conjugated secondary antibody. The amount of total protein was measured by biotinylation and visualization with a streptavidin-Cy 5 conjugate. The results are summarized in Table 1.

Intramembrane variability was expressed as standard deviation of signal intensities (as a percentage of mean intensity). Variability of signal intensities of total protein was $23 \%$ across a P-FILM stack as compared to an average of $19.8 \%$ for specific proteins. For traditional dot blot, intramembrane variability was $17 \%$ for total protein and $19 \%$ for the specific proteins. Intermembrane variability of signal intensity through the five-membrane P-FILM stack generated in one transfer was 1.01-fold for total protein and an average of 1.87-fold for specific proteins. Variability of signal intensity between five nitrocellulose membranes generated by five different transfers was 0.64 -fold for total protein and an average of 1.58 -fold for the specific proteins. Due to the higher throughput of the assay and increased savings of time and sample, we find MLDot to be a viable alternative to traditional blotting (despite slightly higher variability of P-FILM when compared to nitrocellulose membrane).

\section{Influence of Protein Concentration on MLDot Analysis}

We next determined the optimal linear range of protein binding by the membranes to allow quantitative comparison of the samples. Since higher amounts of protein should result in lower intermembrane variability but may saturate the membranes, it is crucial to balance intermembrane variability with the linear range of protein binding. We performed a titration experiment of MLDot with different amounts of Jurkat protein ranging from $0.5 \mu \mathrm{g} /$ well to $80 \mu \mathrm{g} / \mathrm{well}$ in quadruplicate through a five-membrane P-FILM stack and visualized either total protein (Figure 1A) or the specific proteins c-Myc and c-Raf (Figure 1, B and C) on all of the membranes.

The dependence of signal intensity on the amount of input protein is shown on the graphs in Figure 1, right panels. A predicted curve was plotted based on an arbitrary reference point (the intensity of the upper left dot) and the assumption that there is a proportional dependence of signal intensity on the amount of input protein. We determined $2.5-20 \mu \mathrm{g}$ of input protein as the loading interval in which the slopes of the curves generated from experimental data 
points were equivalent to the slope of the predicted curve for detection of total proteins (Figure 1A), c-Myc (Figure 1B), and c-Raf (Figure 1C). Thus, to compare expression levels of selected proteins between samples by MLDot, an initial concentration of $2.5-20 \mu \mathrm{g}$ of input protein is optimal. However, if less then $5 \mu \mathrm{g}$ of protein is loaded, then fewer membranes can be generated. In contrast, higher amounts of input sample (40-80 $\mu \mathrm{g})$ may be used to generate a greater number of replicate blots that have lower intermembrane variability but yield more qualitative data. Therefore, 15-20 $\mu \mathrm{g}$ is the recommended amount of input protein to provide the optimal balance between maintaining the ability to detect concentration changes in a linear fashion and achieving reasonably low intermembrane variability.

The recommended size of proteins to be tested by MLDot is $10-200 \mathrm{kDa}$. We have used MLDot to successfully detect over 45 nuclear, cytoplasmic, and membrane proteins within this size range (data not shown). Furthermore, the P-FILM membranes have been used to successfully detect over 40 proteins (ranging from 30-140 kDa) in a gel-based application (http://www.2020gene.com). Overall, the P-FILM membranes show optimal performance for detecting proteins between 30 and $120 \mathrm{kDa}$.

To compare MLDot with traditional dot blot, we repeated the titration experiment but transferred proteins to five nitrocellulose membranes by performing five traditional dot blot transfers. Signal intensities on nitrocellulose membranes show greater deviation from the standard curve as well as greater variability between membranes, especially for total protein and c-Raf (Figure 2, A and C). As with MLDot, signal

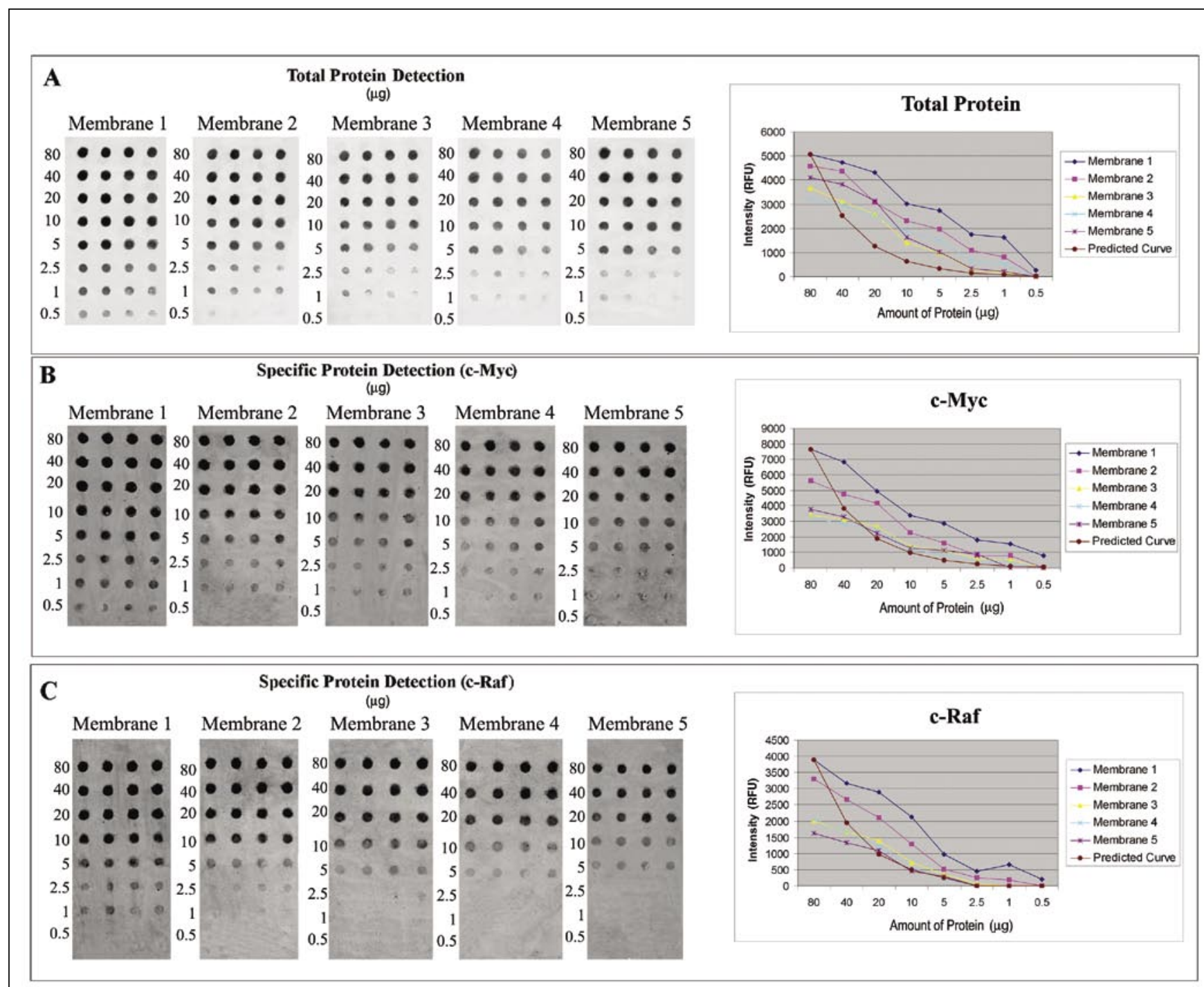

Figure 1. Influence of protein concentration on protein binding to P-FILM membranes. (A) Detection of total protein after biotinylation/streptavidin-Cy5 detection. The graph represents a correlation between signal intensity and amount of protein loaded. (B and C) Detection of c-Myc protein (B) and c-Raf protein (C) on five membranes by using anti-c-Myc antibody or anti-c-Raf antibody and fluorescein isothiocyanate (FITC) detection. P-FILM, protein function and identification layered membranes; RFU, relative fluorescence unit. 
intensities of c-Myc followed the predicted curve between 2.5 and $20 \mu \mathrm{g}$ of input protein (Figure 2B). However, with input protein of $20 \mu \mathrm{g} / \mathrm{sample}$, five membranes can be generated by MLDot in one experiment in contrast to a single membrane generated by dot blot. Therefore, MLDot saves time and sample as compared to traditional dot blot. Furthermore, P-FILM membranes show a more reproducible linear binding range than nitrocellulose membranes.

\section{Profiling Proteome Changes in Activated Human Peripheral Blood Lymphocytes by Using MLDot}

To assess the applicability of MLDot to a biological system, we profiled proteome changes in activated human peripheral blood lymphocytes in response to multiple combinations of T-cell mitogens. MLDot was performed, and the multivariate data from the immunoblot intensities were analyzed by principal component analysis (PCA). PCA profiles trends in complex data sets that examine changes of many variables under multiple conditions. The PCA output is a $3-\mathrm{D}$ plot that clusters the proteomic changes in $3-\mathrm{D}$ space as determined by the average summation of how the different mitogen combinations influenced the signal intensity. The mitogenic response for each proteomic change is clearly distinguished and phospho-tyrosine signals (purple) cluster much higher in the vertical dimension (principal component 2 or PC2) (Figure 3A). Moreover, the results (performed in triplicate with three independent experiments represented by each data point) are highly reproducible. A plot of the eigenvector contribution to the vertical dimension (PC2) (Figure $3 \mathrm{~B}$ ) reveals two expected results: ( $i)$ the mitogen stimulus most responsible for the phospho-tyrosine proteomic

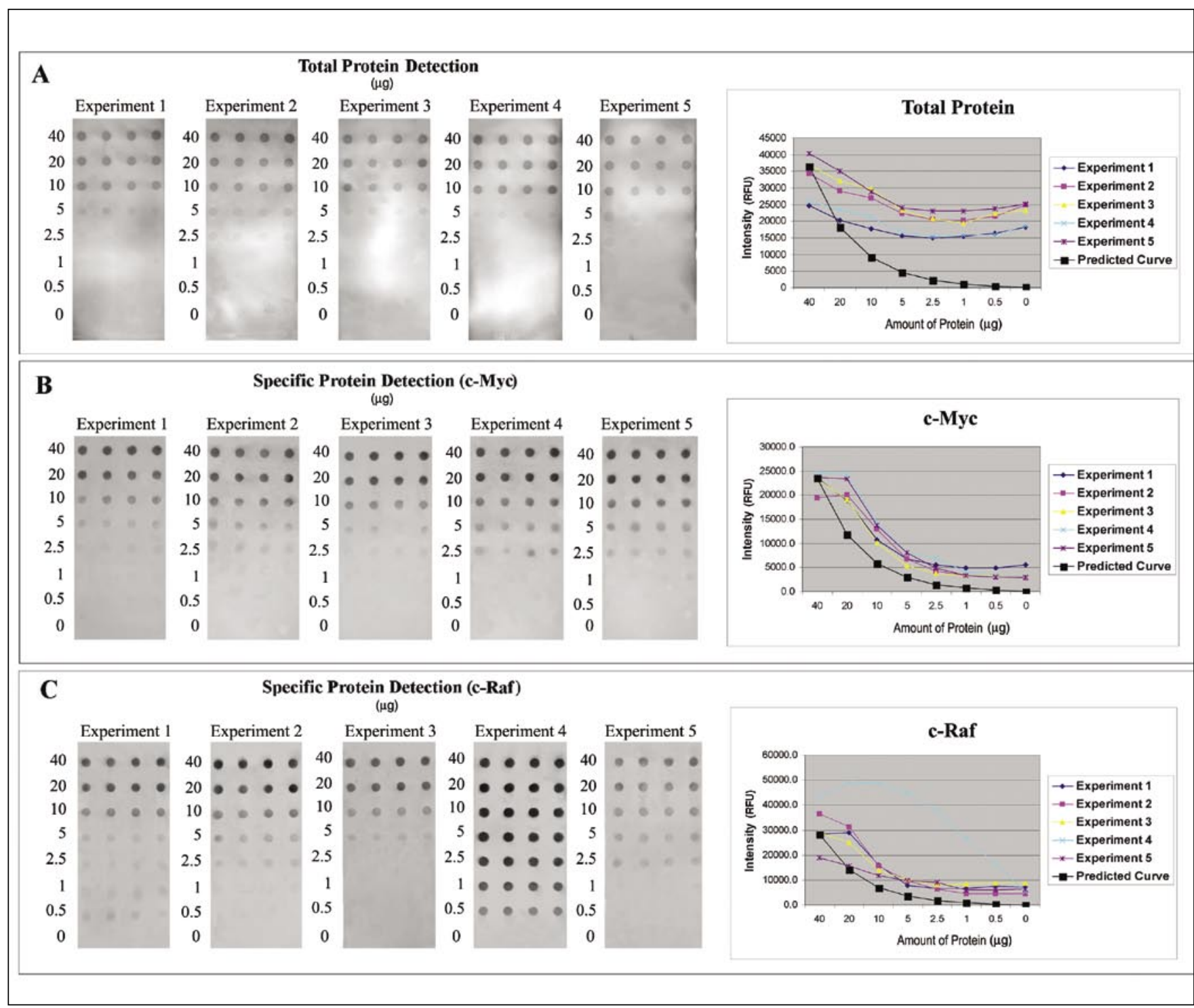

Figure 2. Influence of protein concentration on protein binding to nitrocelluose membranes. (A) Detection of total protein after biotinylation/streptavidin-Cy5 detection. The graph represents a correlation between signal intensity and amount of protein loaded. (B and C) Detection of c-Myc protein (B) and c-Raf protein (C) on five membranes by using anti-c-Myc antibody or anti-c-Raf antibody and fluorescein isothiocyanate (FITC) detection. RFU, relative fluorescence unit. 


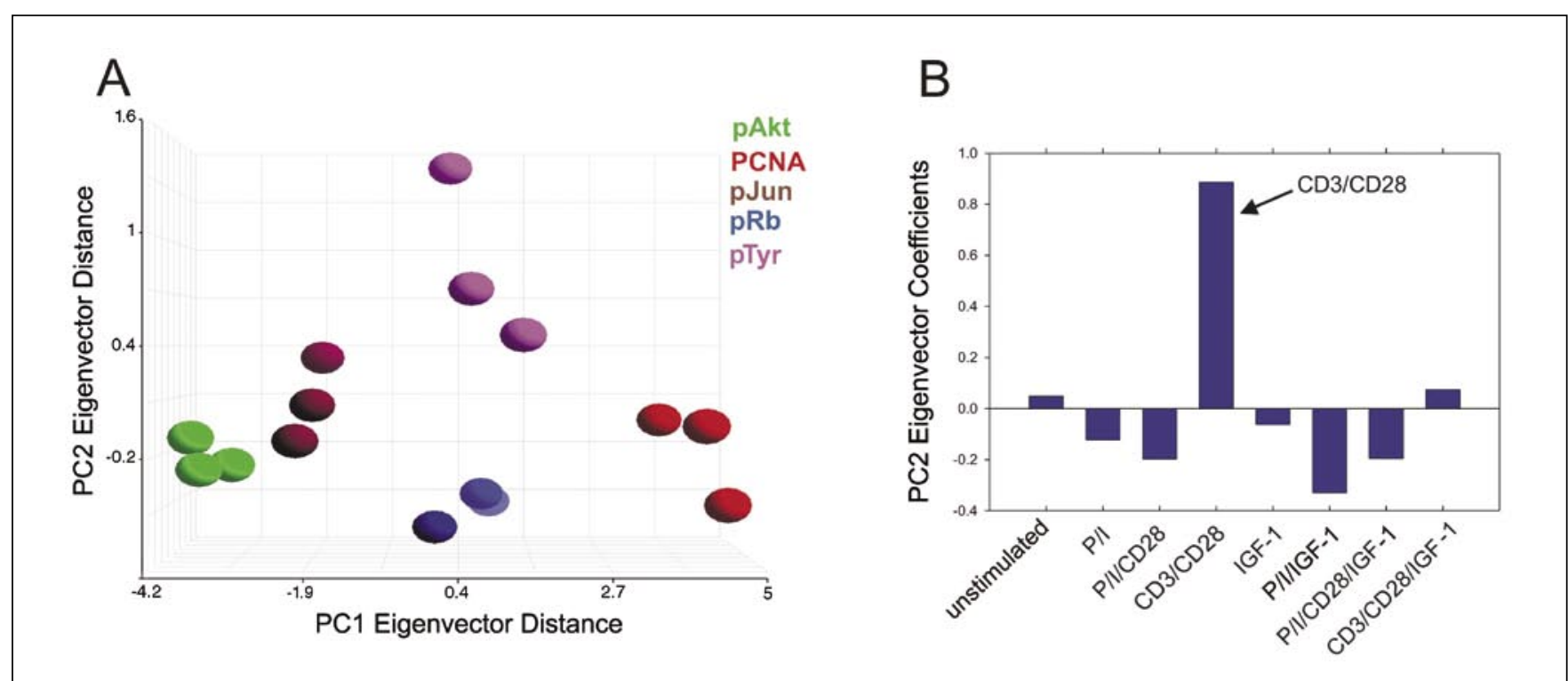

Figure 3. PCA of proteomic profiles in mitogen-stimulated primary human T cells. (A) Human peripheral blood lymphocytes were treated with the indicated mitogen combinations as indicated in Materials and Methods and Figure 3B. Lysates were analyzed for expression of phospho-Akt (pAkt; green), PCNA (red), phospho-Jun (pJun; brown), phospho-Rb (pRb; blue), and phospho-tyrosine (pTyr; purple) by MLDot. The data for each point was generated from the mean of triplicate independent lysates and determinations, and each point represents an independent experiment. Clustering indicates similarities in the mitogen response signature for each antigen. PC1 and PC2 eigenvector distances are in relative units of variance. (B) A plot of the eigenvector coefficients from the PCA output in panel A maps the mitogen contributions to the proteomic signature. The PCA analysis captured 98\% of the variance of the samples. PCA, principal component analysis; MLDot, multilayer multiwell plate dot blotting system; PCNA, proliferating cell nuclear antigen; Rb, retinoblastoma; P/I, phorbol myristate acid/ionomycin; IGF-1, insulin-like growth factor 1; PC1 and PC2, principal components 1 and 2, respectively.

shift is the addition of the cross-linking antibodies for CD3 and CD28 (these receptors initiate tyrosine phosphorylation cascades in activated T cells) and (ii) a much lower contribution to the phospho-tyrosine proteomic shift by mitogen combinations containing phorbol myristate acid/ionomycin (P/I; P/I bypasses the tyrosine phosphorylation cascade in $\mathrm{T}$ cells). Therefore, these data provide proof of principle that MLDot can detect the predicted proteomic changes and show the high reproducibility of MLDot. Interestingly, these data also suggest new information that IGF-1-mediated negative feedback signals may down-regulate tyrosine phosphorylation cascades in T cells.

In summary, MLDot is a technically simple and cost-effective method that increases the throughput of traditional dot blot. In contrast to technologies that can profile one sample for multiple proteins or multiple samples for one protein, MLDot provides the ability to easily quantitate the amounts of multiple proteins in multiple samples simultaneously. Thus, MLDot provides an excellent approach for quantitative proteomic analyses for smaller laboratories.

\section{ACKNOWLEDGMENTS}

M.M.G. and J.L.T. contributed equally to this work.

\section{REFERENCES}

1.Elia, G., M. Silacci, S. Scheurer, J. Scheuermann, and D. Neri. 2002. Affinity-capture reagents for protein arrays. Trends Biotechnol. 20:S19S22.
2.Vignali, D.A. 2000. Multiplexed particle-based flow cytometric assays. J. Immunol. Methods 243:243-255.

3.Zhu, H., M. Bilgin, and M. Snyder. 2003. Proteomics. Annu. Rev. Biochem. 72:783-812.

4.Patterson, S.D. 2003. Proteomics: evolution of the technology. BioTechniques 35:440-444.

5.Englert, C.R., G.V. Baibakov, and M.R. Emmert-Buck. 2000. Layered expression scanning: rapid molecular profiling of tumor samples. Cancer Res. 60:1526-1530.

6.Chuaqui, R.F., R.F. Bonner, C.J. Best, J.W. Gillespie, M.J. Flaig, S.M. Hewitt, J.L. Phillips, D.B. Krizman, et al. 2002. Post-analysis followup and validation of microarray experiments. Nat. Genet. 32(Suppl):509514.

7.Tangrea, M.A., M.J. Flaig, A. Ramesh, C.J. Best, G.V. Baibakov, S.M. Hewitt, C.D. Mitchell, D.P. Hartmann, et al. 2003. Layered expression scanning: multiplex analysis of RNA and protein gels. BioTechniques 35:1280-1285.

8.Smith, J.L., I. Collins, G.V. Chandramouli, W.G. Butscher, E. Zaitseva, W.J. Freebern, C.M. Haggerty, V. Doseeva, et al. 2003. Targeting combinatorial transcriptional complex assembly at specific modules within the interleukin-2 promoter by the immunosuppressant SB203580. J. Biol. Chem. 278:41034-41046.

9.Knezevic, V., C. Leethanakul, V.E. Bichsel, J.M. Worth, V.V. Prabhu, J.S. Gutkind, L.A. Liotta, P.J. Munson, et al. 2001. Proteomic profiling of the cancer microenvironment by antibody arrays. Proteomics 1:12711278 .

10.Knezevic, V. and M.R. Emmert-Buck. 2003. Proteomic profiling of the cancer microenvironment, p. 309-318. In M. Conn (Ed.), Handbook of Proteomic Methods. Humana Press, Totowa.

Address correspondence to Vladimir Knezevic, 20/20 GeneSystems, Inc., 9700 Great Seneca Highway, Rockville, MD 20850,USA.e-mail:vknezevic@2020gene.com 